NASA/TM-2004-212909

\title{
An Experimental Investigation of Unsteady Thrust Augmentation Using a Speaker-Driven Jet
}

Daniel E. Paxson and Mark P. Wernet

Glenn Research Center, Cleveland, Ohio

Wentworth T. John

Ohio Aerospace Institute, Brook Park, Ohio 
Since its founding, NASA has been dedicated to the advancement of aeronautics and space science. The NASA Scientific and Technical Information (STI) Program Office plays a key part in helping NASA maintain this important role.

The NASA STI Program Office is operated by Langley Research Center, the Lead Center for NASA's scientific and technical information. The NASA STI Program Office provides access to the NASA STI Database, the largest collection of aeronautical and space science STI in the world. The Program Office is also NASA's institutional mechanism for disseminating the results of its research and development activities. These results are published by NASA in the NASA STI Report Series, which includes the following report types:

- $\quad$ TECHNICAL PUBLICATION. Reports of completed research or a major significant phase of research that present the results of NASA programs and include extensive data or theoretical analysis. Includes compilations of significant scientific and technical data and information deemed to be of continuing reference value. NASA's counterpart of peerreviewed formal professional papers but has less stringent limitations on manuscript length and extent of graphic presentations.

- TECHNICAL MEMORANDUM. Scientific and technical findings that are preliminary or of specialized interest, e.g., quick release reports, working papers, and bibliographies that contain minimal annotation. Does not contain extensive analysis.

- CONTRACTOR REPORT. Scientific and technical findings by NASA-sponsored contractors and grantees.
- CONFERENCE PUBLICATION. Collected papers from scientific and technical conferences, symposia, seminars, or other meetings sponsored or cosponsored by NASA.

- SPECIAL PUBLICATION. Scientific, technical, or historical information from NASA programs, projects, and missions, often concerned with subjects having substantial public interest.

- TECHNICAL TRANSLATION. Englishlanguage translations of foreign scientific and technical material pertinent to NASA's mission.

Specialized services that complement the STI Program Office's diverse offerings include creating custom thesauri, building customized databases, organizing and publishing research results ... even providing videos.

For more information about the NASA STI Program Office, see the following:

- Access the NASA STI Program Home Page at http://www.sti.nasa.gov

- E-mail your question via the Internet to help@sti.nasa.gov

- Fax your question to the NASA Access Help Desk at 301-621-0134

- Telephone the NASA Access Help Desk at 301-621-0390

- Write to:

NASA Access Help Desk

NASA Center for AeroSpace Information 7121 Standard Drive

Hanover, MD 21076 
NASA/TM-2004-212909

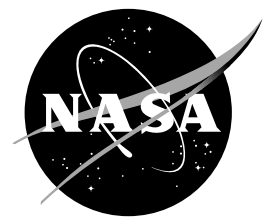

\section{An Experimental Investigation of Unsteady Thrust Augmentation Using a Speaker-Driven Jet}

Daniel E. Paxson and Mark P. Wernet

Glenn Research Center, Cleveland, Ohio

Wentworth T. John

Ohio Aerospace Institute, Brook Park, Ohio

Prepared for the 42nd Aerospace Sciences Meeting and Exhibit sponsored by the American Institute of Aeronautics and Astronautics Reno, Nevada, January 5-8, 2004

National Aeronautics and Space Administration

Glenn Research Center 
This report is a formal draft or working paper, intended to solicit comments and ideas from a technical peer group.

This report contains preliminary findings, subject to revision as analysis proceeds.

This work was sponsored by the Low Emissions Alternative Power Project of the Vehicle Systems Program at the NASA Glenn Research Center.

Available from

NASA Center for Aerospace Information 7121 Standard Drive

Hanover, MD 21076
National Technical Information Service 5285 Port Royal Road Springfield, VA 22100 


\title{
AN EXPERIMENTAL INVESTIGATION OF UNSTEADY THRUST AUGMENTATION USING A SPEAKER-DRIVEN JET
}

\author{
Daniel E. Paxson ${ }^{*}$ and Mark P. Wernet ${ }^{\dagger}$ \\ National Aeronautics and Space Administration \\ Glenn Research Center \\ Cleveland, Ohio 44135 \\ Wentworth T. John* \\ Ohio Aerospace Institute \\ Brook Park, Ohio 44142
}

\begin{abstract}
An experimental investigation is described in which a simple speaker-driven jet was used as a pulsed thrust source (driver) for an ejector configuration. The objectives of the investigation were twofold: First, to add to the experimental body of evidence showing that an unsteady thrust source, combined with a properly sized ejector generally yields higher thrust augmentation values than a similarly sized, steady driver of equivalent thrust. Second, to identify characteristics of the unsteady driver that may be useful for sizing ejectors, and predicting what thrust augmentation values may be achieved. The speaker-driven jet provided a convenient source for the investigation because it is entirely unsteady (having no mean component) and because relevant parameters such as frequency, time-averaged thrust, and diameter are easily variable. The experimental setup will be described, as will the various measurements made. These include both thrust and Digital Particle Imaging Velocimetry of the driver. It will be shown that thrust augmentation values as high as 1.8 were obtained, that the diameter of the best ejector scaled with the dimensions of the emitted vortex, and that the so-called Formation Number serves as a useful dimensionless number by which to characterize the jet and predict performance.
\end{abstract}

\section{INTRODUCTION}

Unsteady ejectors have received renewed attention in recent years, primarily due to the heightened interest in pulse detonation-based propulsion systems, which produce unsteady, impulsive thrust. It has been demonstrated in the past $^{1,2}$ and recently ${ }^{3-5}$ that properly dimensioned ejectors, driven by unsteady thrust sources, often produce higher levels of thrust augmentation, at a considerably smaller size than do those driven by steady jets.

For reference, thrust augmentation is defined as follows:

$$
\phi \equiv \frac{\overline{\mathrm{T}}^{\text {Total }}}{\overline{\mathrm{T}}^{\mathrm{j}}}
$$

where $\overline{\mathrm{T}}^{\text {Total }}$ is the total time-averaged thrust of the ejector and driver and $\overline{\mathrm{T}}^{\mathrm{j}}$ is the time-averaged thrust of the jet alone.

The impetus for the experiment described in this paper was the observation that the recent demonstrations (experiments) referenced were inconsistent in their peak performance. A variety of thrust sources were utilized in Refs. 3-6; however, all shared a similar ejector style (nearly cylindrical). Parameters of the ejectors (length, diameter, inlet leading edge radius) were varied in each experiment to achieve peak thrust augmentation levels, which will be referred to in this paper as geometrical optimization. It was observed however that marked differences in peak augmentation levels were obtained with the different experiments. Even the same

\footnotetext{
*Senior Member AIAA, Phone: 216-433-8334;

Fax: 216-433-8643; E-mail: Daniel.E.Paxson@nasa.gov

†Phone: 216-433-3752; Fax: 216-433-8000;

E-mail: Mark.P.Wernet@nasa.gov

‡Phone: 216-433-3750; Fax: 216-433-8000;

E-mail: Wentworth.T.John@nasa.gov
} 


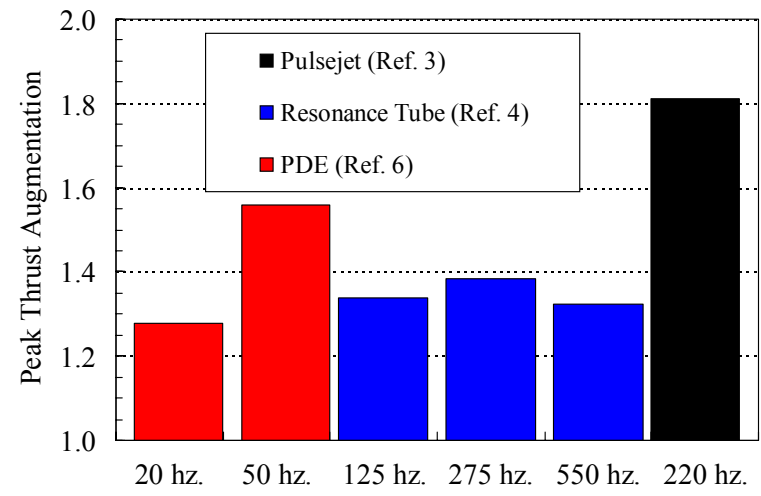

Fig. 1 Peak thrust augmentation levels achieved with various drivers using geometrically optimized, nearly cylindrical ejectors. Colors indicate driving source. Categories indicate operating frequency.

source operating at different frequencies yielded different augmentation levels. ${ }^{*}$ This is shown in Fig. 1, where the peak thrust augmentation achieved for the various thrust sources are plotted, along with their respective operational frequencies. The obvious conclusion to be drawn from the above observation is that the presence of unsteadiness alone does not guarantee superior performance. There are characteristics of the unsteady jet which produce better or worse performance. Subsequent Digital Particle Imaging Velocimetry (DPIV) of both the Ref. 3 pulsejet $^{9}$ and Ref. 4 resonance tube suggested that one critical feature of the flow is the vortex which is emitted with each pulse of the driver. Such vortices can be seen for each of the Refs. 3 and 4 drivers in Figs. 2 and 3, respectively. The figures show ensemble-averaged, instantaneous vorticity contours at the approximate moment when the emitted vortex would enter an ejector. Although not present when the PIV measurements were made, outlines of the ejectors yielding peak thrust augmentation are shown in each figure. In each case the optimal ejector is precisely sized to just bound the emitted vortex. This striking observation served to substantiate the notion that the starting vortex plays a vital role in the performance of unsteady ejectors, and that parameters which characterize the vortex may

\footnotetext{
"The Ref. 6 PDE (Pulse Detonation Engine) experiment was not geometrically optimized as were those of Refs. 3-5. Instead a single ejector was used with a diameter-to-jet diameter ratio similar to both the optimized Ref. 3-5 experiments. This is consistent with the optimal findings of Refs. 7-8, where a similar sized PDE was used as a driver. The length used was the longest available at the time of the experiment. The inlet radius was also the largest available, a choice which is consistent with the Ref. 3 experiment.
}

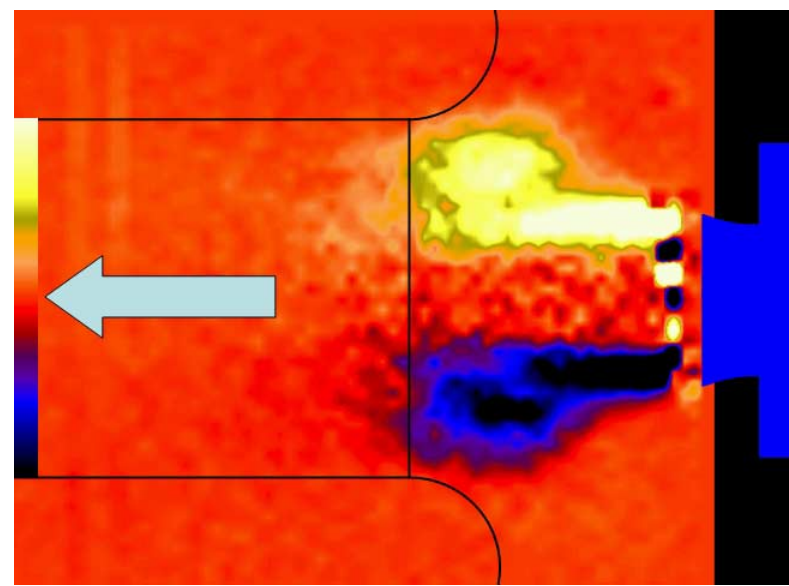

Fig. 2 Color contours of 150 sample, ensembleaverged, instantaneous vorticity in a pulsejet-driven flow. The measurements were made using DPIV. An outline of the ejector profile yielding peak thrust augmentation is shown but was not present during velocity measurements.

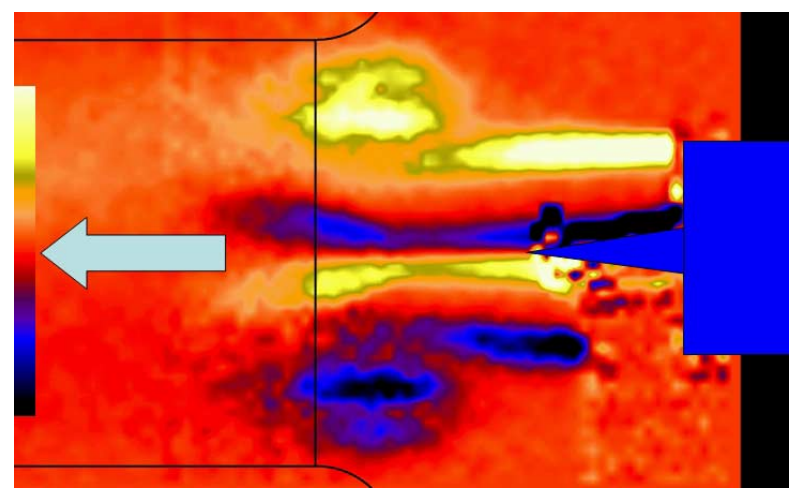

Fig. 3 Color contours of 200 sample, ensembleaverged, instantaneous vorticity in a resonance tube-driven flow. The measurements were made using DPIV. An outline of the ejector profile yielding peak thrust augmentation is shown but was not present during velocity measurements.

characterize the ejector performance as well. One such parameter is the so-called Formation Number. ${ }^{10}$ Its proposed use in predicting geometrically optimized ejector performance is briefly reviewed below from Ref. 3. The thrust from an unsteady driver can be divided into a steady and an unsteady (but periodic) component. The thrust augmentation can similarly be divided. From this division the total thrust augmentation can be expressed as 


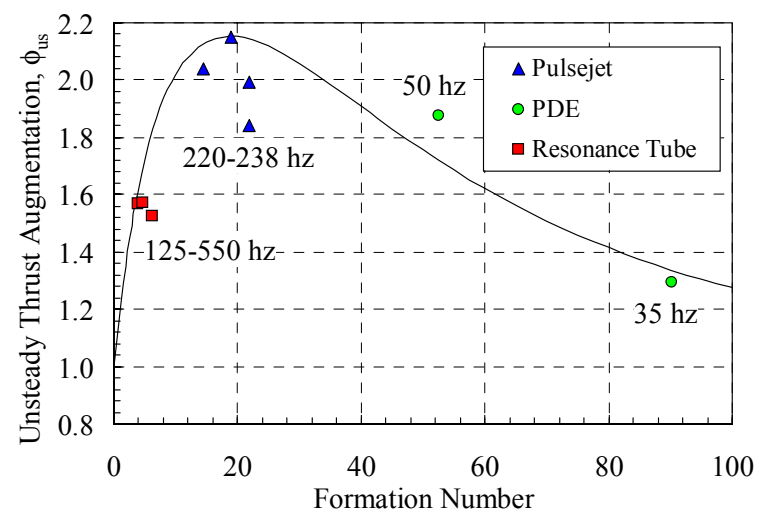

Fig. 4 Unsteady Thrust Augmentation for optimized ejector/driver configurations using a variety of sources: Resonance tube (red), Pulsejet (green and blue, PDE (black). The smooth curve represents heuristic modeling of $\beta_{u s}$ and $\eta_{u s}$, fit to available data, and used in Eq. 3.

$$
\phi=\frac{\phi_{\mathrm{ss}}+\left(\frac{\overline{\mathrm{u}^{\prime 2}}}{\overline{\mathrm{u}}^{2}}\right) \phi_{\mathrm{us}}}{1+\left(\overline{\frac{\mathrm{u}^{\prime 2}}{\overline{\mathrm{u}}^{2}}}\right)}
$$

where the jet velocity $\mathrm{u} \equiv \overline{\mathrm{u}}+\mathrm{u}^{\prime}$, and where, if $T$ is the thrust, then $\phi_{\mathrm{ss}}=\frac{\overline{\mathrm{T}}_{\mathrm{ss}}^{\text {Total }}}{\overline{\mathrm{T}}_{\mathrm{ss}}^{\mathrm{j}}}$ and $\phi_{\mathrm{us}}=\frac{\overline{\mathrm{T}}_{\mathrm{us}}^{\text {Total }}}{\overline{\mathrm{T}}_{\mathrm{us}}^{\mathrm{j}}}$. Here, overbars represent time averages, the superscript $j$ refers to the jet alone, and the superscript Total refers to the jet/ejector combination. The steady state augmentation component can be estimated using available correlations or mixing calculations. The unsteady thrust augmentation component, it is proposed, can be written in the form

$$
\phi_{\mathrm{us}}=\left(1+\beta_{\mathrm{us}}\right)^{\frac{\eta_{\mathrm{us}}}{2}}
$$

where $\beta_{\mathrm{us}}$, and $\eta_{\mathrm{us}}$ are the unsteady entrainment ratio (secondary to primary mass flow) and efficiency respectively. This is a similar form to a model used for steady ejectors. ${ }^{11}$ Both of the parameters in Eq. 3, and therefore the unsteady thrust augmentation itself, are thought to be sole functions of the jet Formation Number. ${ }^{10}$ In this work, the Formation Number (an inverse Strouhal Number or reduced frequency) is defined as

$$
F=\frac{\sqrt{\overline{\mathrm{u}^{\prime 2}}}}{2 \mathrm{fd}_{\mathrm{j}}}
$$

where $f$ is the frequency of the primary source, and $\mathrm{d}_{\mathrm{j}}$ is the jet diameter. ${ }^{\dagger}$ It is noted that $\overline{\mathrm{u}^{\prime 2}}$ is impossible to measure in some experiments, but can be inferred from thrust data, or estimated from simulations. With $\beta_{\text {us }}$ a monotonically increasing function of $F$ and $\eta_{\text {us }}$ flat to some critical value of $F$, then monotonically decreasing (based on limited data from Refs. 3 and 4), the form of $\phi_{\text {us }}$ becomes that shown in Fig. 4. Also shown in this figure are the available results measured from several experiments. The comparison between predictions and experiment is encouraging, particularly with regard to the basic shape. There appears to be a particular formation number at which the best unsteady performance is found, with performance falling off rapidly to the left and gradually to the right. The data however, is admittedly sparse. The sparse nature of the data was, in fact, the impetus for the experiment to be described.

If an unsteady thrust source could be developed with the capability of large variations in frequency, $\overline{\mathrm{u}^{\prime 2}}$, and jet diameter, then a range of Formation Numbers could be examined. This could be used to validate the notion of Formation Number as a performance metric since it could be varied in several ways (e.g. diameter, frequency, and $\overline{\mathrm{u}^{\prime 2}}$ ).

A speaker-driven, or so-called synthetic, jet meets these requirements and has the added benefit of having no steady velocity component (zero net mass flow), thereby isolating the thrust augmentation to only the unsteady component.

\footnotetext{
${ }^{\dagger}$ Equation 4 is a slightly different form than Ref. 10 from which it was obtained. In Ref. 10, the velocity and time scales were presented as $\int_{0}^{t_{\text {outflow }}} \mathrm{u} d t$, where $t_{\text {outflow }}$ denotes the time period over which the flow is leaving the jet source. This is often a difficult quantity to obtain, and may not be appropriate when the unsteady jet source has a large steady component of velocity. In jets where the steady velocity component is small, it is probable that the integral above is closely related to $\frac{\sqrt{\overline{\mathrm{u}^{\prime 2}}}}{2 \mathrm{f}}$.
} 

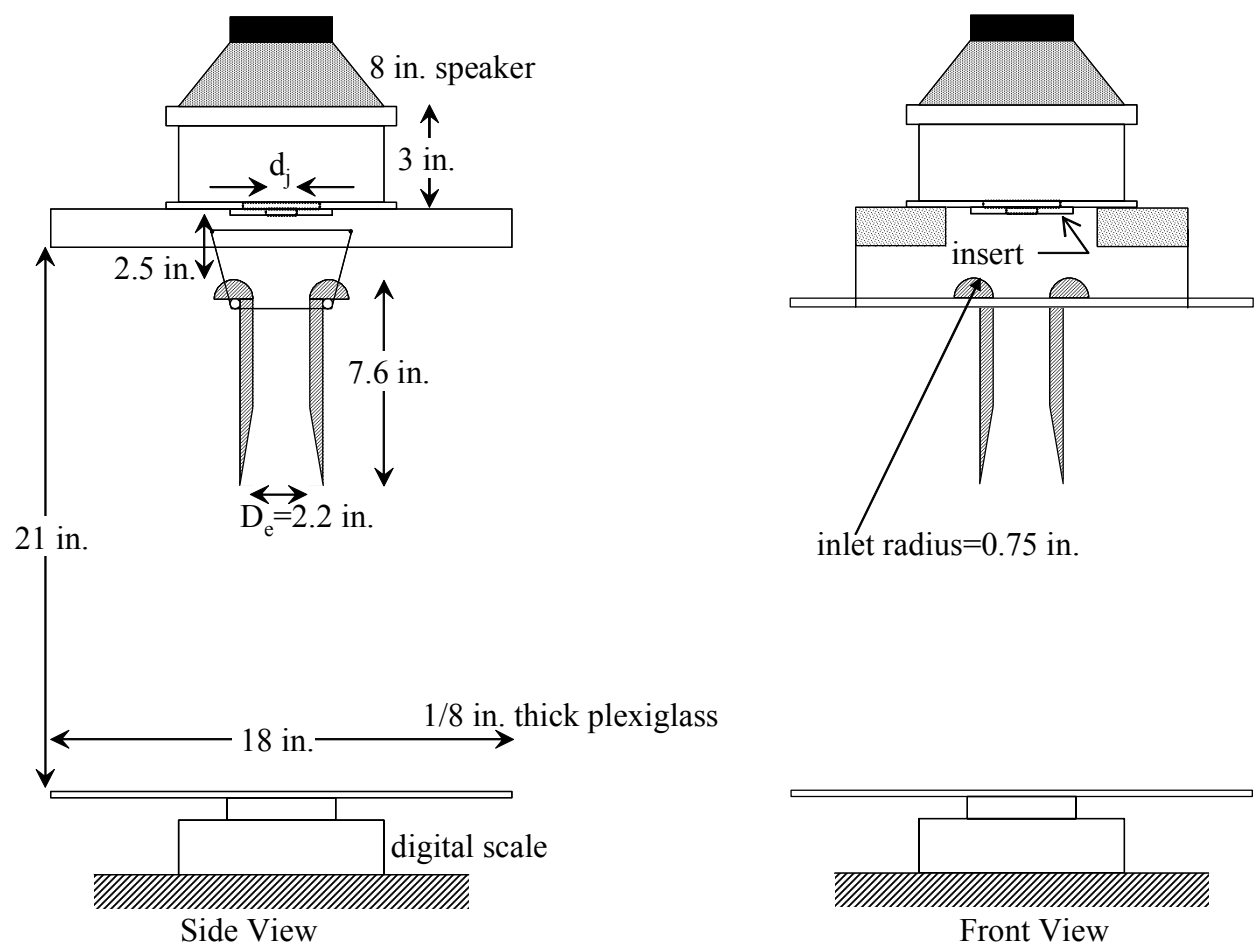

Fig. 5 Experimental setup and nomenclature for the speaker-driven jet.

One potential drawback to the device is that the thrust levels and corresponding velocities are exceptionally low, possibly putting the flow in a different regime from the other devices examined. This will be discussed in subsequent sections.

This paper describes the speaker-driven jet experiment and presents the results obtained to date. It will be shown that Formation Number as defined above is an excellent correlating parameter (i.e. much of the data collapses onto a single curve when it is plotted as a function of $F$ ). It will also be shown however, that it is not the only required parameter. There are apparently additional characteristics of the jet flow that impact performance since the peak thrust augmentation levels achieved with the speaker driven jet were below those seen in Fig. 1. Possible explanations will be offered.

Additionally, limited results from particle imaging velocimetry (PIV) measurements obtained on the system will be presented to supplement discussions and assumptions. No description of the PIV system or method will be presented as it is well described elsewhere?.

\section{EXPERIMENTAL SETUP}

The experimental arrangement is shown to scale in Fig. 5, along with relevant nomenclature. An 8 inch, dual coil speaker (Peerless brand) was mounted on the back of a cylindrical cavity. The other end of the cavity was sealed except for an exit hole, the diameter of which could be varied by means of the inserts shown. The speaker was driven by an Altec/Lansing Model 1269 audio amplifier. The input signal for the amplifier was a sinusoid of variable frequency supplied by a Wavetek Model 193 signal generator. The speaker and cavity arrangement was mounted on a support stand from which an ejector could be suspended. Below this an 18 in. square plexiglass plate was mounted on a Mettler-Toledo Model PB5001-S digital scale (with 0.1 gram accuracy). The scale rested on the floor of the laboratory. All measurements of thrust were made by simply reading the output on the scale. This was the only method available, and was sub-optimal due to the fact that readings were often fluctuating. A longduration, time-averaging procedure would have been preferable; however, given the large number of readings obtained, it is believed that the overall error introduced by the method used is small. Output from the audio amplifier was measured on a Tektronix Model TDS-220 oscilloscope from which both frequency and rms voltage could be obtained. 


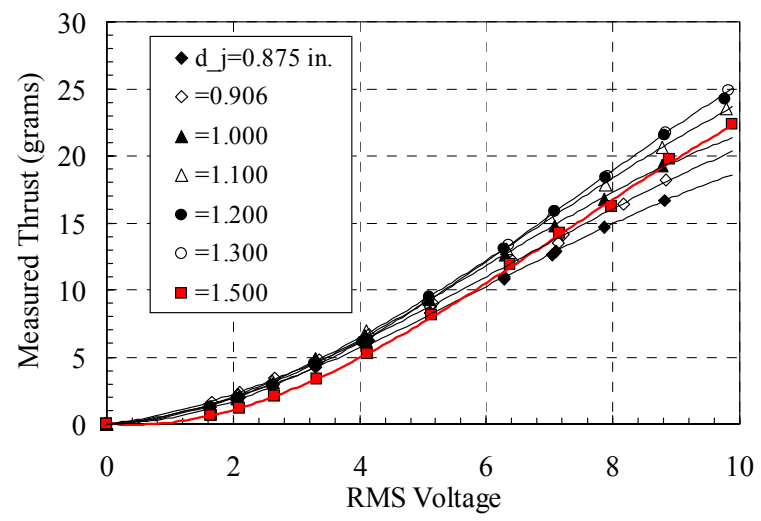

Fig. 6 Measured time-averaged jet thrust as a function of RMS speaker voltage for various jet diameters at $20 \mathrm{hz}$. excitation.

\section{JET CHARACTERIZATION}

The arrangement was initially tested with no ejector in place so that the denominator of Eq. 1 could be determined. Typical results are shown in Fig. 6 where measured thrust is plotted against rms voltage for various jet diameters, at a driving frequency of $20 \mathrm{hz}$. All of the data appears smooth and is well fit using a cubic polynomial for each diameter. It is observed that for a given rms excitation, the thrust increases with increasing jet diameter until $\mathrm{d}_{\mathrm{j}}=1.5 \mathrm{in}$. It is not clear why this 'peak' occurs; however, it is consistent with the observation that at extremely large jet diameters $\left(d_{j}>4\right.$ in.) virtually no thrust was observed. The upper limit of excitation voltage was determined by estimating the safe power limits of the speaker. Because this thrust reduction was observed with jet diameters at and above 1.5 in., thrust augmentation data was collected only for jet diameters smaller than this.

Figure 7 shows measured thrust plotted against rms voltage for various frequencies, at a jet diameter of 1.2 in. Here it is seen that thrust obtained for a given excitation is essentially independent of frequency from $20<\mathrm{f}<50$ but falls off substantially above or below this range. At low frequency, the thrust reduction appears to be related to the speaker coil reaching fixed travel limits. The reason for the high frequency reduction is not clear. It may be related to the coil response, or it may be a fluid mechanical effect. It is noted that at approximately $200 \mathrm{hz}$. there is no measurable thrust. Whatever the mechanism behind the changes in thrust for a given excitation, the frequencies at which they occurred were deemed as limits for augmentation testing. Thus, augmentation results (i.e. thrust with an ejector) were only collected at frequencies between 15 and $70 \mathrm{hz}$.

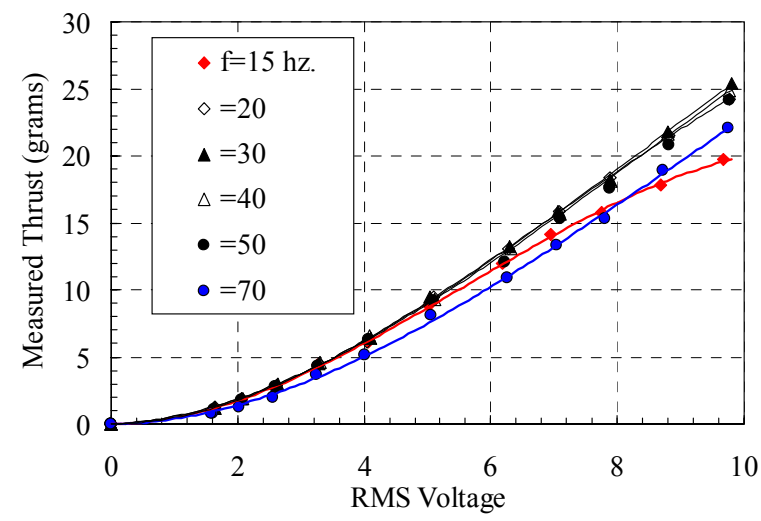

Fig. 7 Measured, time-averaged jet thrust as a function of RMS speaker voltage for various excitation frequencies at $d_{j}=1.2$ inches.

\section{AUGMENTATION}

Only one ejector diameter was tested in this experiment. Due to the peculiarities of the setup, it was much easier to obtain geometric optimization by varying the driver jet diameter than by varying the ejector diameter as was done in Refs. 3 and 4. The ejector inlet radius (see Fig. 5) was set at 0.75 in. and no testing was done to determine an optimal value (previous experiments indicate that the effect is small and in general, the bigger the better). Because it was difficult to change the position of the ejector, all augmentation measurements were made at a driver-to-ejector inlet spacing of approximately $2.5 \mathrm{in}$. This effect is also expected to be fairly small based on previous results.

For each jet diameter and frequency, thrust as a function of rms voltage was first determined for the jet alone and fit to a cubic least squares approximation. The ejector was then put in place and thrust (now total) was again measured against excitation voltage. From this data, and the cubic fit, thrust augmentation was determined.

Thrust augmentation as a function of the rms excitation voltage is shown in Fig. 8 for a range of jet diameters. The ejector length is $7.6 \mathrm{in}$. The data was obtained at an excitation frequency of $20 \mathrm{hz}$. In general, a jet diameter of 1.2-1.3 inches yielded the highest augmentation over the range of excitation voltage. Similar results were found at frequencies of 30 and $40 \mathrm{hz}$. The next largest jet diameter insert available was 1.5 in. which, as noted in Fig. 6 produced somewhat anomalous results and was not used for augmentation studies. Thus, the 1.2 in. jet was chosen as being geometrically optimized for the ejector and was used in subsequent tests to vary Formation Number. This diameter jet was also used in tests to determine the 


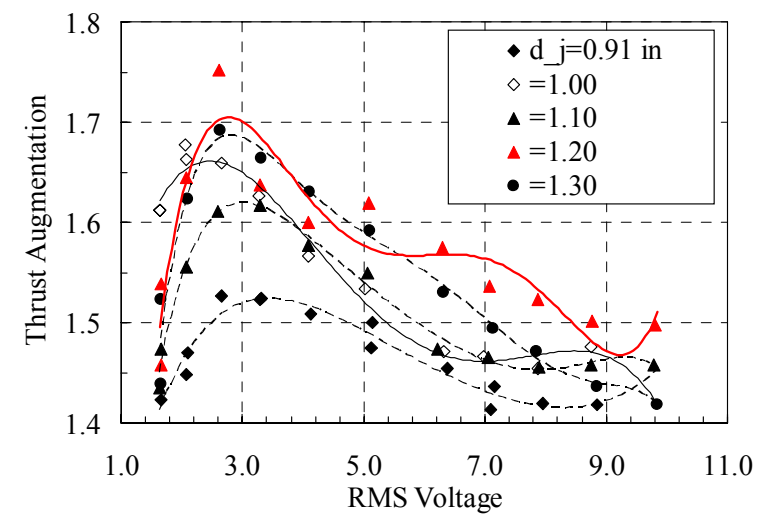

Fig. 8 Measured, time-averaged Thrust Augmentation as a function of the RMS speaker voltage for various jet diameters at an excitation frequency of $20 \mathrm{hz}$.

optimal length of the ejector. Three different length ejectors were tested over a limited set of frequencies Of these, the 7.6 in. length ejector shown in Fig. 5 yielded the best results.

Subsequent PIV measurements on this jet yielded results similar to those noted in Figs. 2 and 3, namely that the boundary of the emitted vortex coincides with the ejector diameter. This is illustrated in Fig. 9 which shows 200 point ensemble-averaged, instantaneous contours of vorticity for the emitted vortex of the $1.2 \mathrm{in}$. jet at a frequency of $50 \mathrm{hz}$. and an rms speaker excitation voltage of 10.0 volts. An outline of the ejector is also shown. Identical images were obtained at $20 \mathrm{hz}$. excitation.

It is observed that ratio of ejector body diameter (2.2 in.) to driver diameter (1.2 in.) yielding optimal performance is quite different, at 1.83 , than those of other unsteady driver experiments, which range from 2.4-3.0. This observation suggests that a speaker-driven vortex may scale in size somewhat differently than one emanating from a 'pipe' such as a pulsejet, resonance tube, or pulse detonation engine. This will be discussed in the next section of the paper.

\section{CALCULATION OF FORMATION NUMBER}

The Formation Number defined by Eq. 4 requires an rms velocity, which generally must be inferred or computed based on available data. A momentum balance will show that for a speaker driven jet with no net mass flow, the time-averaged thrust is

The ejectors used here were constructed for use in another experiment. The lengths and diameters available were limited.

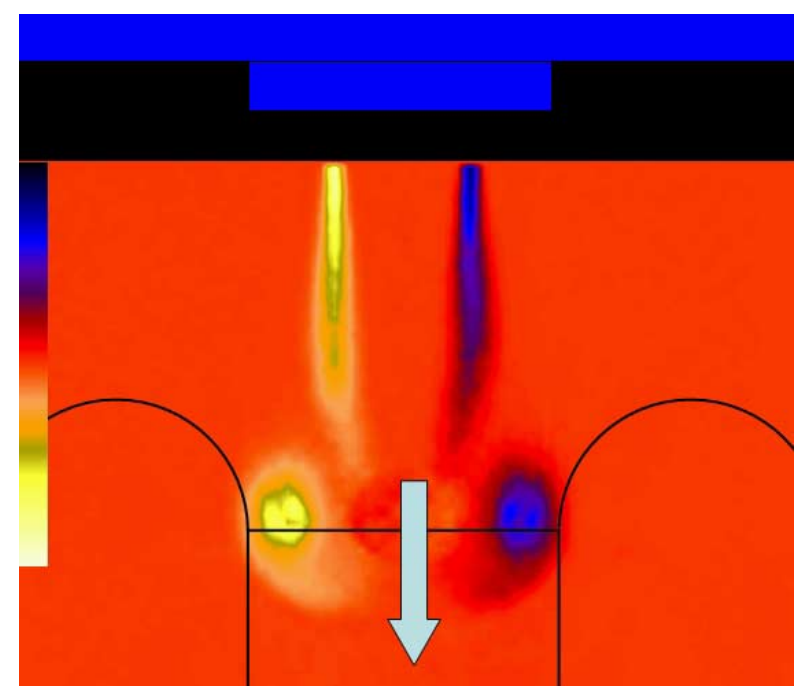

Fig. 9 Color contours of 200 sample, ensembleaverged, instantaneous vorticity in a speakerdriven flow. The measurements were made using DPIV. An outline of the ejector profile yielding peak thrust augmentation is shown but was not present during velocity measurements.

$$
\overline{\mathrm{T}}^{\mathrm{j}}=\left(\frac{\rho \mathrm{A}}{\mathrm{g}_{\mathrm{c}}}\right) \overline{\mathrm{u}^{\prime 2}}+\frac{\mathrm{A}}{\tau_{\text {cycle }}} \int_{0}^{\tau_{\text {cycle }}}\left(\mathrm{p}_{\mathrm{e}}-\mathrm{p}_{\mathrm{amb}}\right) \mathrm{d} \tau
$$

where $A$ is the jet area, $\mathrm{p}_{\mathrm{e}}$ is the static pressure at the jet exit plane, and $p_{a m b}$ is the ambient pressure. During outflow periods it may be assumed that $\mathrm{p}_{\mathrm{e}}=\mathrm{p}_{\mathrm{amb}}$. During inflow periods it is estimated that $\mathrm{p}_{\mathrm{e}}-\mathrm{p}_{\mathrm{amb}} \approx-\frac{\rho \overline{\mathrm{u}^{\prime 2}}}{2 \mathrm{~g}_{\mathrm{c}}}$. Thus, with inflow occurring over approximately half the cycle

$$
\overline{\mathrm{T}}^{\mathrm{j}} \cong 0.75\left(\frac{\rho \pi \mathrm{d}_{\mathrm{j}}^{2}}{4 \mathrm{~g}_{\mathrm{c}}}\right) \overline{\mathrm{u}^{\prime 2}}
$$

Equations 5 and 6 assume a spatially uniform distribution of pressure and rms velocity across the jet; however, and it was observed in the PIV measurements that this was not the case. This is clearly seen in Fig. 10 which shows the measured distributions of rms velocity for two different sized jets, at two different Formation Numbers. Each point is a time-average of 16 phaselocked instants comprising a full cycle. Each instant consists of 200 ensemble-averaged measurements. The measurements are $0.2 \mathrm{in}$. downstream of the jet exit. The physical boundaries of the jet openings are marked 


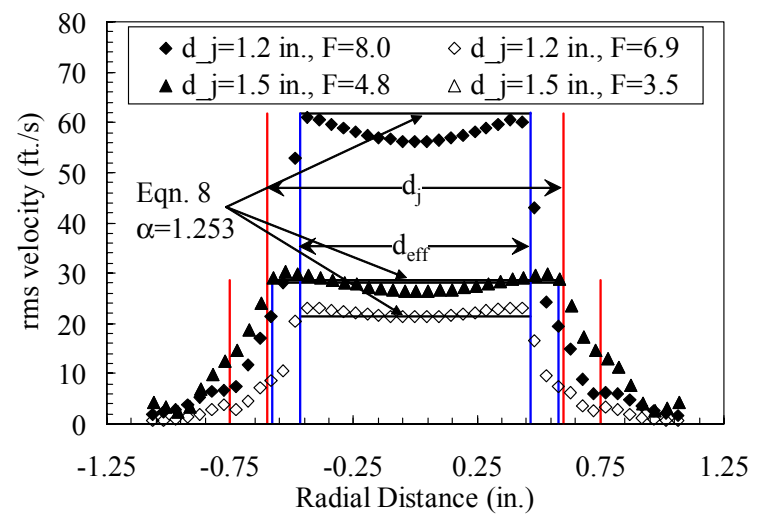

Fig. 10 RMS velocity as a function of radial distance for several jets obtained using PIV. Each point is an average of 16 phase locked instants consisting of 200 ensemble averaged measurements. The measurements are 0.2 in. downstream of the exit.

in with red lines. All of the rms distributions appear to have a nearly uniform region that is smaller than the physical jet diameter. Since it is this 'core' flow that is of importance in vortex formation, it seems reasonable to characterize the jet not by the physical diameter or area, but by an effective diameter, and to use the core rms velocity fluctuations to determine Formation Number. For both of the jets measured it was found that

$$
\mathrm{d}_{\mathrm{eff}}=\mathrm{d}_{\mathrm{j}} \sqrt{0.6}
$$

Interestingly, this corresponds closely to a typical discharge coefficient for a sharp edged orifice in steady flow. It is also interesting to note that, using this effective diameter makes the ratio of ejector to (effective) jet diameter at which peak performance occurs 2.4 , which is nearly identical to that found with the pulsejet-driven system. That is to say it reinforces the growing evidence that optimal unsteady ejectors scale in diameter with the vortex, and that the vortex scales in size with the effective jet source.

In light of the observed non-uniformities in rms velocity, the observed presence of an effective diameter, and the fact that it is an estimate, Eq. 6 was modified, and rearranged to solve for rms velocity as

$$
\sqrt{\overline{\mathrm{u}^{\prime 2}}}=\frac{1}{\mathrm{~d}_{\text {eff }}}\left(\frac{\overline{\mathrm{T}}^{\mathrm{j}} 4 \mathrm{~g}_{\mathrm{c}}}{\alpha \rho \pi}\right)^{1 / 2}
$$

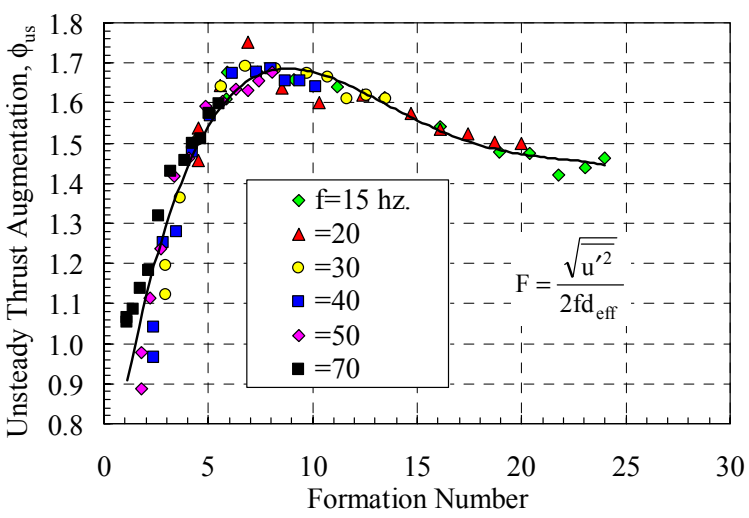

Fig. 11 Thrust Augmentation as a function of the Formation Number with $d_{j}=1.2$ in. and $D_{e}=2.2$ in. Formation Number has been varied using both frequency and rms velocity.

where it is understood that the rms velocity referred to is the jet core value. The coefficient $\alpha$ was determined for each of the four jet velocity fields measured using PIV and the corresponding measured thrust values. Averaging the four $\alpha$ values yielded a final result of $\alpha=1.253$, with a standard deviation of $7 \%$. The rms velocity values calculated with Eq. 8 and this $\alpha$ for the four jets measured with PIV are shown in Fig. 10.

\section{RESULTS}

Formation Number, as described above, and thrust augmentation, were obtained for the 1.2 in. diameter jet and 2.2 in. diameter ejector over a range of frequencies from 15 to $70 \mathrm{hz}$., and over a range of jet thrust levels from 0 to 25 grams. The data was used to make the plot shown in Fig. 11. Here, Thrust Augmentation (unsteady by definition) is plotted as a function of jet Formation Number for the entire frequency and thrust range tested. The fact that all of the data collapse neatly on a single curve is strong evidence that the Formation Number of Eq. 4 is indeed a relevant parameter for predicting geometrically optimized ejector performance. The curve shown in Fig. 11 is simply a polynomial fit to the data, however, it is remarkably similar in shape to that formed by Eq. 3 and its underlying assumptions (i.e., entrainment increases with $\mathrm{F}$, and efficiency is initially flat then decreasing with F). This result lends a measure of credence to such a modeling approach.

\section{DISCUSSION}

While Fig. 11 demonstrates a consistent relationship between Formation Number and Thrust Augmentation for the speaker-driven jet, it is somewhat inconsistent with results obtained using other unsteady data. 


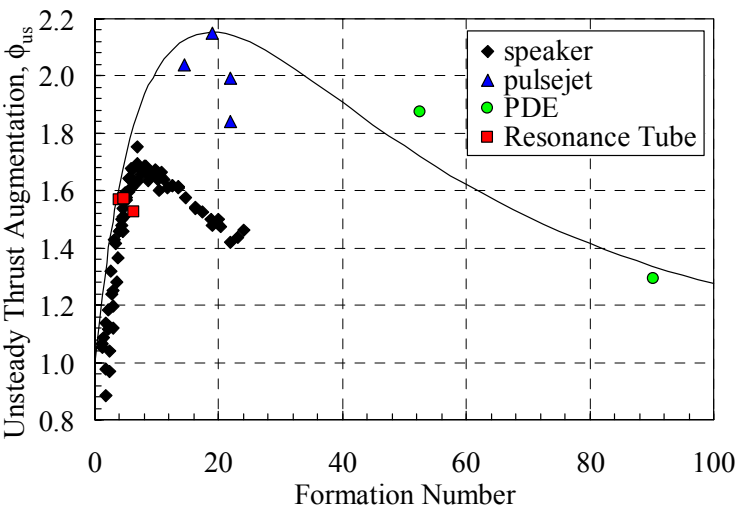

Fig. 12 Unsteady Thrust Augmentation as a function of the Formation Number for all tested thrust sources.

Figure 12 combines the results of Figs. 4 and 11 onto a single plot. Here it is seen that while the speaker-driven system has the same trend as the other systems, the peak values are not as high and the Formation Number at which the peak occurs seems to be somewhat lower.

With regard to the latter discrepancy, it should be kept in mind that the values of $\sqrt{\overline{\mathrm{u}^{\prime 2}}}$ used in calculating Formation Numbers of the other experiments are estimated from equations similar to Eq. 6. While this proved accurate for the speaker-driven jet, it may not prove reliable for the other thrust sources. Subsequent PIV measurements on the resonance tube and pulsejet sources for example showed values of $\sqrt{\overline{\mathrm{u}^{\prime 2}}} / \overline{\mathrm{u}}$ to be approximately 0.83 and 1.74 , respectively. These were somewhat less than the estimated values of 1.04 and 1.89. This result reduces the Formation Number (but raises the unsteady Thrust Augmentation) for both cases. Furthermore, in the case of the Pulsejet and PDE, the density (used in Eq. 6) of the emitted flow could not be measured. Estimates based on simulations were used, and these may have inaccuracies too.

Regarding the differences in peak thrust augmentation values between the present and previous experiments, several mechanisms seem plausible and will be discussed below.

\section{Reynolds Number}

The vorticity transport equations (being derived from the Navier-Stokes equations) yield a dissipation term that is preceded by a Reynolds Number. For the unsteady jets under consideration here, an appropriate Reynolds Number may be written:

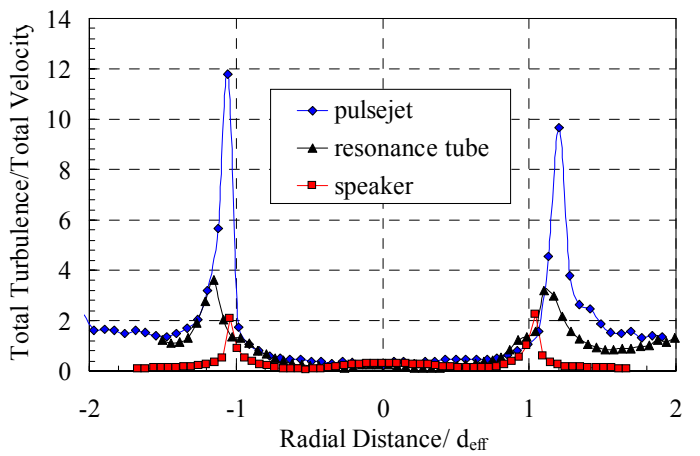

Fig. 13 Total Turbulence distributions for three separate pulsed thrust sources. Turbulence has been scaled by local total ensemble-averaged velocity. Radial distance has been scaled by effective diameter. The measurements were made using PIV at the approximate downstream location where the vortex enters the ejector.

Table 1 Reynolds Number estimates for Several Pulsed Thrust Experiments.

\begin{tabular}{|c|c|c|c|c|}
\hline & $\begin{array}{c}\text { Speaker- } \\
\text { Driven }\end{array}$ & Pulsejet & $\begin{array}{c}\text { Resonance } \\
\text { Tube }\end{array}$ & PDE \\
\hline $\mathrm{R}_{\mathrm{e}}$ & $3.0 \times 10^{4}$ & $3.5 \times 10^{4}$ & $2.5 \times 10^{5}$ & $1.8 \times 10^{5}$ \\
\hline
\end{tabular}

The typical values for the four experiments are shown in Table 1. Although the pulsejet has rms velocities that are an order of magnitude above the speaker-driven system, the emitted flow is much hotter and therefore much less dense, and much more viscous. The result is that the two systems have comparable Reynolds numbers but much different performance. Conversely, the speaker-driven and resonance-tube systems have vastly different Reynolds numbers, but similar performance. It is therefore unlikely that this is the explanation for the differences; however, it is noted that the length and velocity scales used to define the Reynolds Number shown may not be appropriate.

Turbulence

Here again, the impact is unclear; however, it is noted that the pulsejet, which showed the highest thrust augmentation, has a vortex which is considerably more turbulent than the others for which such measurements were possible. Figure 13 shows the instantaneous, ensemble-averaged, total Turbulence distributions for three separate pulsed thrust sources. Turbulence has 


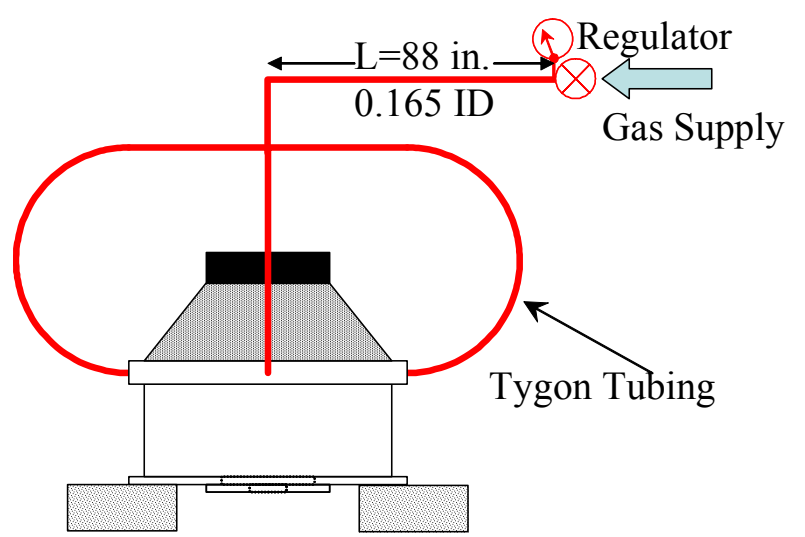

Fig. 14 Schematic diagram of modifications to the speaker-driven jet allowing for forced addition of a supplementary gas. The lines (in red) are distributed symmetrically about the cavity circumference.

been scaled by local, instantaneous, ensemble-averaged, total velocity. Note that the turbulence shown is distinct from the periodic velocity fluctuation used in calculating Formation Number. Radial distance has been scaled by effective diameter. The measurements were made at the approximate downstream location where the vortex enters the ejector. Of note in the figure is the observation that the regions of high vorticity are the same as those of high turbulence and that the pulsejet driven flow has particularly high values. It is possible that the turbulence in this region acts as an effective mechanism by which the vortex transfers its rotational energy to the entrained flow. Alternately, the turbulence may act to enhance entrainment of secondary flow by the vortex.

It is probable that the high turbulence level of the pulsejet flow result from the violent combustion processes taking place within the device. It is probable that these are present in the PDE as well, which is consistent with the relatively high augmentation results observed.

It is interesting to note that preliminary computational simulations of a PDE-driven ejector system similar to the one actually tested ${ }^{6}$, but using an inviscid code yielded no thrust augmentation ${ }^{12}$. The addition of laminar viscosity may make little difference to such a calculation; however, high levels of turbulent viscosity may have a substantial impact.

\section{Emitted Flow Enthalpy}

It is observed that the pulsed sources with the highest emitted enthalpy seem to yield the highest unsteady thrust augmentation. No explanation can be offered as to why this is so. In fact, steady ejector results indicate a reduction in performance as the enthalpy increases relative to the entrained flow.

As a simple test of this observation suitable to the current speaker-driven system, the setup shown in Fig. 5 was modified such that a steady flow of a low molecular weight gas could be introduced into the cavity region. The modified setup is shown schematically in Fig. 14. The gas chosen was helium. The jet diameter was 1.2 in. The concept for such a system was that ambient air would enter the cavity on the 'in-stroke' of the speaker, mix with whatever helium was forced into the cavity, and exit on the 'out-stroke' as a uniform mixture at some intermediate density and specific enthalpy. No provision was made to validate this vision. Nor, due to time constraints, was any provision made to measure the enthalpy of the emitted gas.

Although no flow meter was available, a pressure regulator at the gas supply point, followed by 88 inches of 0.165 in. ID tubing allowed for reasonable estimates using a Moody Chart. ${ }^{13}$

Because of the limited supply of helium in the facility, testing was extremely limited. Only one frequency was tested. The value chosen was $15 \mathrm{hz}$. At this frequency only two speaker excitation voltages were tested. These were rms voltages of 2.63 and $8.79 \mathrm{~V}$.

The jet was first tested alone at various levels of auxiliary gas injection using both air and helium. The two gases were used in order to distinguish between changes in thrust due to using a light (high specific enthalpy) gas and those due to auxiliary injection itself. The results are shown in Fig. 15. Here, the measured jet thrust is plotted as a function of estimated auxiliary gas volumetric flow rate for both helium and air as auxiliary gases, using the two different excitation voltages. Each point is an average of several readings. The estimated auxiliary flow rate has been normalized by the product of the effective area and the rms velocity of the jet with no auxiliary gas present (Eq. 8). Values of this normalized flow rate that are greater than 1 roughly indicate that the auxiliary gas flow completely fills the added chamber volume during the intake stroke of the speaker. That is, there is no longer inflow through the jet opening. Also shown in the figure (as open symbols), for air only, are the expected thrust levels if the estimated steady flow components were simply added to the unsteady component when there is no injection.

Several features of this plot are surprising, and tend to render the augmentation results which follow somewhat 


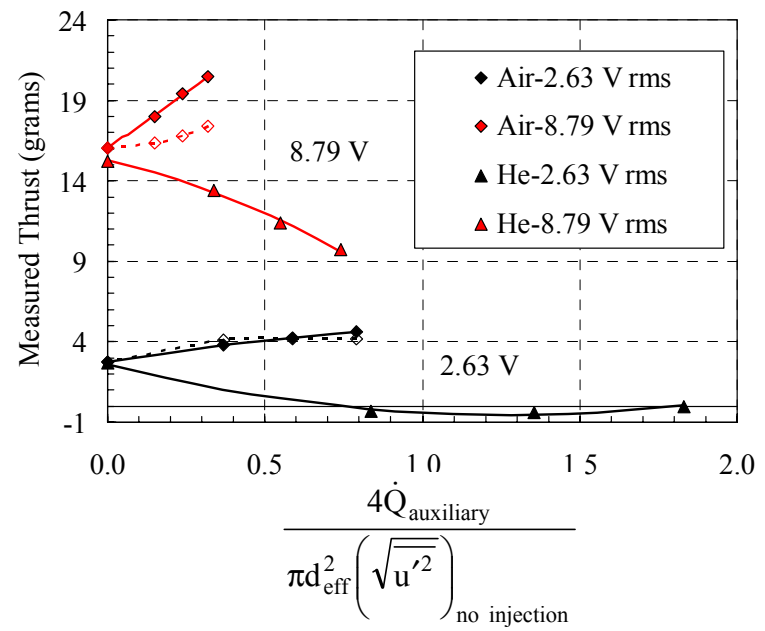

Fig. 15 Measured jet thrust as a function of estimated auxiliary gas flow rate for the modified speaker-driven jet at two excitation voltage levels, using auxiliary helium and air. The frequency is $15 \mathrm{hz}$. Open symbols represent expected thrust due to the addition of estimated steady air mass flow.

inconclusive, though still worthwhile. Consider first the results with air as auxiliary gas. It was expected that the addition of auxiliary air would lead to an increase in thrust, since it adds a component to Eq. 6. While this seems to hold well for the low-voltage excitation, it does not for the high-voltage case. The latter result suggests that the presence of the auxiliary air has an impact on the motion of the speaker itself and, in turn, on the manner in which fluid moves in and out of the cavity.

A second, and perhaps more striking feature of Fig. 15 is the apparent negative thrust (lift on the thrust plate of Fig. 5) observed in the low-voltage case, with helium as the auxiliary gas. This is believed to be related to buoyancy effects, which can influence the thrust measurements due to the particular orientation of the thrust plate and jet. (i.e., it is pointing downward). Although the mechanism is not clear, the negative thrust may arise when the lightweight gas jet forms a sheet as it is deflected across the thrust plate. Alternately, the gas may never become a sheet. It may begin to spread across the thrust plate, then rise from it. Both situations could give rise to a negative thrust reading. It should be kept in mind as well that at the low speaker excitation levels, the emitted flow is composed entirely of helium (see Fig. 15). Thus, based on density alone, the thrust should be approximately 7 times less than that measured with air as the auxiliary gas. Extrapolating the air results of Fig. 15 to auxiliary flow rates comparable to those with helium would yield a maximum thrust of

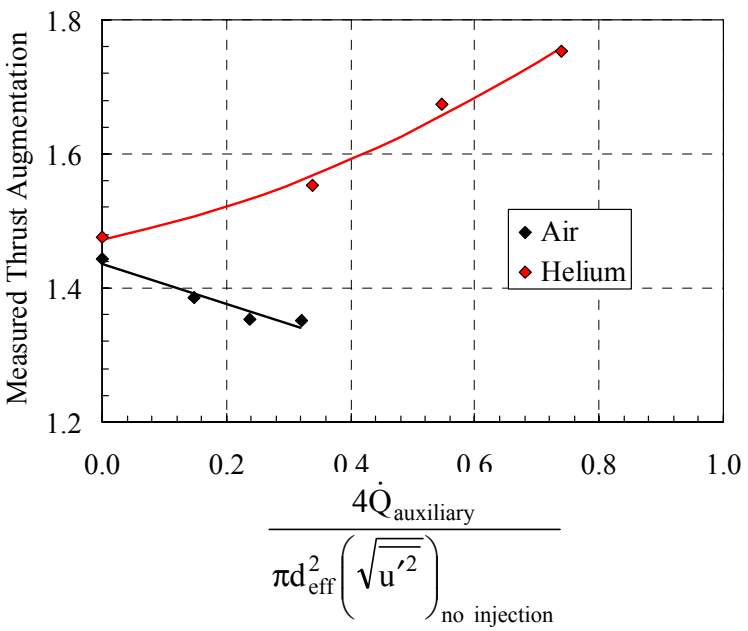

Fig. 16 Measured thrust augmentation as a function of estimated auxiliary gas flow rate for the modified speaker-driven jet at an excitation voltage level of 8.79 V., using auxiliary helium and air. The frequency is $15 \mathrm{hz}$.

only about 1 gram. A small error due to buoyancy could easily lead to negative readings. Additionally, if the emitted flow from the jet is thought of as a series of self contained 'balls' of helium, the deceleration due to their buoyancy could result in them moving significantly slower by the time they reach the plate than when they left the exit hole of the jet. This, in turn would lead to thrust reading even less than the 1.0 gram estimate just given.

Whatever the cause, the low-voltage excitation data was not considered usable, and all of the results shown hence will focus on the high-voltage. It is noted, with respect to the high-voltage excitation data, that the decrease in thrust observed with the helium auxiliary gas is consistent with the emitted gas being lighter. Bouyancy effects are expected to be lower in this case however, as the emitted gas is not nearly as light as the low voltage case. Furthermore, the thrust levels are much higher, and the errors, even if the same as the low voltage case, are a considerably smaller fraction of the total.

Thrust augmentation results for the high-voltage case are presented in Fig. 16. Like Fig. 15, data is plotted as a function of estimated auxiliary gas volumetric flow rate. The trend of the auxiliary air data is expected in light of Eq. 2. As more gas is injected the steady component of thrust rises. The thrust augmentation due to this steady component is considerably less than the unsteady component, and thus the overall augmentation should decrease. The trend for auxiliary helium data 


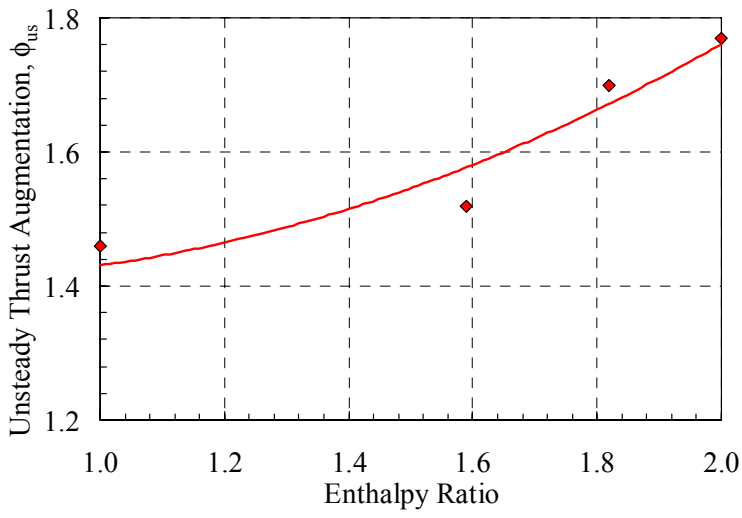

Fig. 17 Derived unsteady thrust augmentation as a function of jet enthalpy ratio for the modified speaker-driven jet at an excitation voltage level of 8.79 V., using auxiliary helium. The frequency is $15 \mathrm{hz}$.

seems to support the notion that high enthalpy jet flows improve the unsteady thrust augmentation.

A crude estimate of the jet density and hence specific enthalpy may be obtained as follows. Assuming that thrust may be calculated as

$$
\overline{\mathrm{T}}^{\mathrm{j}} \cong\left(\frac{\rho \pi \mathrm{d}_{\mathrm{eff}}^{2}}{4 \mathrm{~g}_{\mathrm{c}}}\right)\left(\overline{\mathrm{u}^{\prime 2}}+\overline{\mathrm{u}}^{2}\right)
$$

For the jet with Auxiliary air injection, $\rho$, and $\overline{\mathrm{u}}$ are known (the latter from the estimated auxiliary flow rate). Equation 10 can thus be used to obtain $\overline{\mathrm{u}^{\prime 2}}$. This value was a fairly constant $3652 \mathrm{ft}^{2} / \mathrm{s}^{2}$ for the three air injection cases tested, with only $3.8 \%$ standard deviation. Assuming this same value of $\overline{\mathrm{u}^{\prime 2}}$ for the helium auxiliary gas and noting that

$$
\dot{\mathrm{m}}_{\mathrm{He}} \approx \frac{\bar{\rho} \overline{\mathrm{u}} \pi \mathrm{d}_{\mathrm{eff}}^{2}}{4}
$$

Equations 10 (with $\rho=\bar{\rho}$ ) and 11 can be solved simultaneously to obtain $\bar{\rho}$ and $\overline{\mathrm{u}}$. These can, in turn be used to obtain the average specific enthalpy ratios (from the properties of helium and air mixtures) and the values of unsteady augmentation from Eq. 2. A steady thrust augmentation estimate (based on a simple constant area mixing calculation) of 1.2 is used. This exercise results in the plot shown in Fig. 17 where unsteady thrust augmentation is plotted as a function of jet enthalpy ratio. It is clear that thrust augmentation increases with increasing enthalpy ratio.

\section{CONCLUSIONS}

The experiment described in this paper has demonstrated that a versatile, small scale, speakerdriven, pulsed thrust system can be used to investigate unsteady thrust augmentation in general. It was verified that the Formation Number, as defined in Eq. 4, is a relevant; though not sufficient parameter on which to base unsteady ejector performance. Furthermore, it was demonstrated that unsteady thrust augmentation, plotted as a function of Formation Number exhibits a maximum value, as predicted by the modeling approach of Eq. 3 . The Formation number at which the maximum occurs is close to that identified in Ref. 10 as marking the point when the emitted vortex no longer contains all of the emitted vorticity. It was also shown that the jet-toambient enthalpy ratio may be another critical parameter in predicting unsteady thrust augmentation.

The DPIV measurements presented strongly suggest that the ejector diameter for which optimal augmentation is obtained is directly linked to geometric size of the vortex ring, and that the vortex ring size is geometrically related to the (effective) jet diameter. In this work, it was found that the optimal ejector diameter is approximately 2.4 times that of the driver. It was also concluded from these measurements that vortex turbulence may play a role in unsteady ejector performance.

\section{REFERENCES}

1. Lockwood, R.M. "Interim Summary Report on Investigation of the Process of Energy Transfer from an Intermittent Jet to Secondary Fluid in an Ejector-Type Thrust Augmenter," Hiller Aircraft Report No. ARD-286, March, 1961.

2. Binder, G. and Didelle, H, "Improvement of Ejector Thrust Augmentation by pulsating or flapping Jets," Paper E3 of Proc. 2nd Symposium on Jet Pumps \& Ejectors and Gas Lift Techniques, Cambridge, England, March 1975.

3. Paxson, D.E., Wilson, J., and Dougherty, K.T., "Unsteady Ejector Performance: An Experimental Investigation Using a Pulsejet Driver," AIAA paper 2002-3915, July, 2002.

4. Wilson, J., and Paxson, D.E., "Unsteady Ejector Performance: An Experimental Investigation Using a Resonance Tube Driver," AIAA paper 20023632, July, 2002.

5. Wilson, J. "A Simple Model of Pulsed Ejector Thrust Augmentation," NASA/CR - 2003-212541, August, 2003. 
6. Paxson, D.E., Unpublished test results using the NASA Glenn 2 Inch Diameter PDE with a Cylindrical Ejector, 2002, also see Thomas, S. Deloof, R., Dougherty, K., "A Review of the NASA Glenn Research Center Pulse Detonation Engine Technology (PDET) Project," presented at 14th Annual Symposium on Propulsion, University Park, PA, December 10-11, 2002.

7. Rasheed, A., Tangirala, V., Pinard, P.F., Dean, A.J., "Experimental and Numerical Investigation of Ejectors for PDE Applications," AIAA paper 20034971, July, 2003.

8. Shehadeh, R., Saretto, S., Lee, S.Y., Pal, S., Santoro, R.J., "Experimental Study of a Pulse Detonation Engine Driven Ejector," AIAA paper 2003-4972, July, 2003.
9. John, W.T., Paxson, D.E., Wernet, M.P., "Conditionally Sampled Pulsejet Driven Ejector Flow Field Using DPIV," AIAA paper 2002-3231, June, 2002.

10. Gharib, M., Rambod, E., Shariff, K., "A universal time scale for vortex ring formation," Journal of Fluid Mechanics, Vol. 360, pp. 121-140, 1998.

11. Porter, J.L., and Squyers, R.A., "A Summary/Overview of Ejector Augmentor Theory and Performance Phase II-Technical Report," ATC Report No. R-91100/9CR-47A, Sept., 1979.

12. Yungster, Shaye, Personal Communications, August, 2003.

13. White, Frank, M., Fluid Mechanics, McGraw-Hill Book Company, 1979. 


\begin{tabular}{|c|c|c|}
\hline \multicolumn{2}{|c|}{ REPORT DOCUMENTATION PAGE } & $\begin{array}{l}\text { Form Approved } \\
\text { OMB No. 0704-0188 }\end{array}$ \\
\hline \multicolumn{3}{|c|}{$\begin{array}{l}\text { Public reporting burden for this collection of information is estimated to average } 1 \text { hour per response, including the time for reviewing instructions, searching existing data sources, } \\
\text { gathering and maintaining the data needed, and completing and reviewing the collection of information. Send comments regarding this burden estimate or any other aspect of this } \\
\text { collection of information, including suggestions for reducing this burden, to Washington Headquarters Services, Directorate for Information Operations and Reports, } 1215 \text { Jefferson } \\
\text { Davis Highway, Suite 1204, Arlington, VA 22202-4302, and to the Office of Management and Budget, Paperwork Reduction Project (0704-0188), Washington, DC 20503. }\end{array}$} \\
\hline 1. AGENCY USE ONLY (Leave blank) & \begin{tabular}{|c|c|} 
2. REPORT DATE & 3. \\
February 2004 &
\end{tabular} & $\begin{array}{l}\text { D DATES COVERED } \\
\text { echnical Memorandum }\end{array}$ \\
\hline \multicolumn{2}{|c|}{$\begin{array}{l}\text { An Experimental Investigation of Unsteady Thrust Augmentation Using a } \\
\text { Speaker-Driven Jet }\end{array}$} & \multirow{2}{*}{ WBS-22-708-90-05 } \\
\hline $\begin{array}{l}\text { 6. AUTHOR(S) } \\
\text { Daniel E. Paxson, Mark P. V }\end{array}$ & et, and Wentworth T. John & \\
\hline $\begin{array}{l}\text { 9. SPONSORING/MONITORING AGEI } \\
\text { National Aeronautics and Sp } \\
\text { Washington, DC 20546-00 }\end{array}$ & $\begin{array}{l}\text { NAME(S) AND ADDRESS(ES) } \\
\text { Administration }\end{array}$ & $\begin{array}{l}\text { 10. SPONSORING/MONITORING } \\
\text { AGENCY REPORT NUMBER } \\
\text { NASA TM-2004-212909 } \\
\text { AIAA-2004-0092 }\end{array}$ \\
\hline
\end{tabular}

Prepared for the 42nd Aerospace Sciences Meeting and Exhibit sponsored by the American Institute of Aeronautics and Astronautics, Reno, Nevada, January 5-8, 2004. Daniel E. Paxson and Mark P. Wernet, NASA Glenn Research Center; and Wentworth T. John, Ohio Aerospace Institute, Brook Park, Ohio 44142. Responsible person, Daniel E. Paxson, organization code 5530, 216-433-8334.

12a. DISTRIBUTION/AVAILABILITY STATEMENT

12b. DISTRIBUTION CODE

Unclassified - Unlimited

Subject Category: 07

Distribution: Nonstandard

Available electronically at http://gltrs.grc.nasa.gov

This publication is available from the NASA Center for AeroSpace Information, 301-621-0390.

13. ABSTRACT (Maximum 200 words)

An experimental investigation is described in which a simple speaker-driven jet was used as a pulsed thrust source (driver) for an ejector configuration. The objectives of the investigation were twofold: first, to add to the experimental body of evidence showing that an unsteady thrust source, combined with a properly sized ejector generally yields higher thrust augmentation values than a similarly sized, steady driver of equivalent thrust. Second, to identify characteristics of the unsteady driver that may be useful for sizing ejectors, and predicting what thrust augmentation values may be achieved. The speaker-driven jet provided a convenient source for the investigation because it is entirely unsteady (having no mean component) and because relevant parameters such as frequency, time-averaged thrust, and diameter are easily variable. The experimental setup will be described, as will the various measurements made. These include both thrust and Digital Particle Imaging Velocimetry of the driver. It will be shown that thrust augmentation values as high as 1.8 were obtained, that the diameter of the best ejector scaled with the dimensions of the emitted vortex, and that the so-called Formation Number serves as a useful dimensionless number by which to characterize the jet and predict performance.

\begin{tabular}{|l|l|l|}
\hline $\begin{array}{l}\text { 14. SUBJECT TERMS } \\
\text { Ejector }\end{array}$ & $\begin{array}{c}\text { 19. SECURITY CLASSIFICATION } \\
\text { OF ABSTRACT } \\
\text { Unclassified }\end{array}$ \\
$\begin{array}{c}\text { 17. SECURITY CLASSIFICATION } \\
\text { OF REPORT } \\
\text { Unclassified }\end{array}$ & $\begin{array}{c}\text { 18. SECURITY CLASSIFICATION } \\
\text { OF THIS PAGE } \\
\text { Unclassified }\end{array}$ &
\end{tabular}

